\section{MS13-P2 A new cubic phase in the \\ Li-Mn-Ge-O system solved by 3D electron diffraction methods}

Holger Klein ${ }^{1,2}$, Christophe Lepoittevin ${ }^{1,2}$, Stéphanie Kodjikian ${ }^{1,2}$, Lei Ding ${ }^{1,2}$, Claire V. Colin ${ }^{1,2}$, Céline Darie ${ }^{1,2}$, Pierre Bordet $^{1,2}$

1. Univ. Grenoble Alpes, Inst NEEL, F-38042 Grenoble, France 2. CNRS, Inst NEEL, F-38042 Grenoble, France

\section{email: holger.klein@neel.cnrs.fr}

Recently, research on quasi-one-dimensional magnetic and multiferroïc materials has renewed the interest in pyroxenes of the stoichiometry $\mathrm{AMX}_{2} \mathrm{O}_{6}(\mathrm{~A}=$ alkali metal, $\mathrm{M}=$ transition metal, $\mathrm{X}=\mathrm{Si}$ or $\mathrm{Ge}$ ). In these phases the magnetic $\mathrm{M}^{3+}$ ions form chains and chemical substitution on the $\mathrm{A}$ and $\mathrm{M}$ sites can change the magnetic coupling along these chains making this system a rich field for the exploration of new phases of interesting magnetic properties [1]. In this work we report the discovery of a new phase in the Li-Mn-Ge-O system. A HP-HT solid state reaction was performed on a mixture of nominal stoichiometry $\mathrm{LiMnGe}_{2} \mathrm{O}_{6}$ during $1 \mathrm{~h}$ at a temperature of $850^{\circ} \mathrm{C}$ and a pressure of $3 \mathrm{GPa}$ in a belt press. Powder X-ray diffraction yielded a diffractogram that could not be indexed by known phases of this system. An electron diffraction study in a transmission electron microscope was conducted in order to identify any unknown phases. In the case of structures that promise interesting properties a more targeted synthesis can then be undertaken. For the purpose of this work, we studied one of several unknown phases in the powder in more detail. From standard selected area electron diffraction the unit cell was determined to be cubic with cell parameter $a=1.17 \mathrm{~nm}$. Precession electron diffraction, eliminating critical multiple diffraction effects, allowed determining the extinction conditions leading to space group Ia3d. EDX analysis in the TEM showed a $\mathrm{Mn} / \mathrm{Ge}$ ratio of $1 / 1$, while the $\mathrm{Li}$ and $\mathrm{O}$ content could not be quantified. Intensities were recorded by in-zone axis precession electron diffraction and by electron diffraction tomography. Combining the data from both methods yielded the structure which we will present here with special attention to the determination of the $\mathrm{Li}$ in this phase.

[1] J. Cheng, W. Tian, J. Zhou et al., 2013, J. American Chem. Soc., 135, 2776-2786

Keywords: electron crystallography, structure determination, pyroxene

\section{MS13-P3 A new fast method to derive \\ Crystallite Size Distributions (CSD) from 2D X-ray diffraction data.}

Sigmund H. Neher ${ }^{1}$, Chaouachi Marwen ${ }^{1}$, Falenty Andrzej ${ }^{1}$, Klein Helmut $^{1}$, Werner F. Kuhs ${ }^{1}$

1. Georg-August-University Göttingen

email: sneher@gwdg.de

We present our new method fast diffraction CSD analysis for deriving CSDs of polycrystalline materials or powders from 2D diffraction data. This analysis can be carried out with standard lab equipment as well as on synchrotron instruments equipped with a $2 \mathrm{D}$ detector. The typical range of application covers crystallite sizes from sub- $\mu \mathrm{m}$ (on synchrotron sources) to hundreds of $\mu \mathrm{m}$.

We will present details of the measurement technique (1), the data reduction (2), the calibration and scaling (3) and (4) a generalized approach to the Lorentz correction, the beam profile correction and the absorption correction.

(1) For the CSD analysis an image sequence of a stepwise rotation measurement (phi or omega rotation) with a "spotty" diffraction patterns is needed. The rotational range is commonly a few degrees with a step size chosen as a fraction of the rocking curve. The appearance of each spot needs to be resolved in a sufficient number of images to evaluate its rocking curve. (2) Data reduction is done by a computer Program written in Python. Every spot is detected, isolated from other overlapping spots (if needed) and assigned to its appearance in consecutive frames. All integrated intensities form the Intensity Distribution (ID) of the sample. (3) For each analysis a standard material with known CSD needs to be measured under exactly the same conditions as the sample. With an established scaling factor between the known standard CSD and the measured standard ID and by using the ratio between the structure factors of sample and standard, the sample CSD can be calculated (see fig.). (4) To both, the sample ID and the standard ID, the Lorentz correction and a absorption correction are applied. In addition the IDs are deconvoluted with the lateral beam profile.

So far the method has been successfully tested with a Bruker APEX II CCD-detector using a laboratory X-ray source, at ESRF ID 15 in Grenoble using the PIXIUM 4700 and with the Perkin Elmer XRD 1622 at DESY Hamburg. For testing we use several well defined single crystal Corundum powder grain size fractions characterized with SEM imagery. A very satisfactory performance was achieved. Due to its speed, the method holds considerable promise also for in-situ studies of crystal growth, recrystallization and coarsening processes [1].

[1] M. Chaouachi et al., In-situ determination of the evolution of the crystallite size distributions of GH-bearing sediments using two-dimensional X-ray diffraction, this conference. 


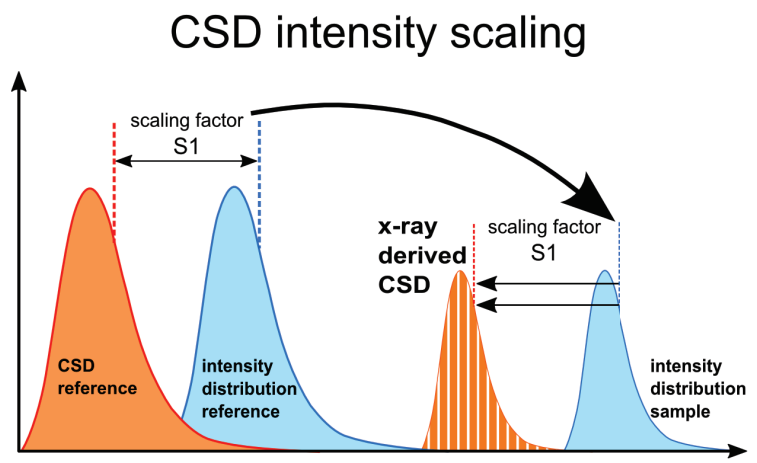

Figure 1. CSD intensity scaling: The scaling factor, S1, between the measured Intensity Distribution (ID) and the volume distribution (CSD) of the reference is used to derive the sample CSD from the measured sample ID.

Keywords: CSD, X-Ray, Crystallite Size Distribution
MS13-P4 Is it possible to collect reliable data on a laboratory diffractometer for a single crystal containing giant disordered supramolecules?

Eugenia V. Peresypkina ${ }^{1,2}$, Alexander V. Virovets ${ }^{1,2}$

1. University of Regensburg, Regensburg, Germany

2. Nikolaev Institute of Inorganic Chemistry SB RAS, Novosibirsk, Russia

email: peresyp@niic.nsc.ru

The advantages of the synchrotron radiation sources in diffraction studies are well-known. But laboratory diffractometers equipped with modern micro-focus X-ray tubes have their advantages, first of all, full-time availability that provides structure determination keeping pace with the ongoing chemical study, and enough space for laboratory equipment to manipulate samples. During last decade we systematically investigate structural chemistry of the compounds based on pentaphosphaferrocene, $\left[\mathrm{Cp}^{\mathrm{R}} \mathrm{Fe}\left(\eta^{5}-\mathrm{P}_{5}\right)\right] \quad\left(\mathrm{Cp}^{\mathrm{R}}=\eta^{5}-\mathrm{C}_{5} \mathrm{R}_{5}\right.$, $\left.\mathrm{R}=\mathrm{Me}, \mathrm{CH}_{2} \mathrm{Ph}\right)$, and $\mathrm{Cu}^{+}, \mathrm{Ag}^{+}\left(\mathrm{M}^{+}\right)$salts. Coordination of $\mathrm{M}^{+}$to $\mathrm{P}$ atoms of cyclo- $\mathrm{P}_{5}$ rings results in various giant supramolecules containing dozens of heavy atoms confined by $\mathrm{Cp}^{\mathrm{R}}$ ligands (Fig. 1) [1,2]. At that, inorganic core is often disordered resulting in weak high-angle reflections. The crystals usually demonstrate strong tendency for twinning and quick loosing of solvent and, therefore, cannot be pre-selected in the lab before transporting to the synchrotron.

Our equipment allows us to keep a sample in the argon atmosphere and select the crystals at about $-100^{\circ} \mathrm{C}$. Using Agilent Technologies SuperNova diffractometer $(\mathrm{CuK} \alpha$ micro-focus source, Titan ${ }^{\mathrm{S}} \mathrm{CCD}$ detector) and single crystals with $\mathrm{V} \sim 30000 \AA^{3}$ we systematically investigate how to measure weak intensities avoiding reflections overlap and how to improve the quality of diffraction data, the reliability of the structural model and quality factors by optimization of such factors as scan width, crystal-to-detector distance and detector mode, exposure time and redundancy. We use the results to tune the data collection strategies originally designed for small molecules and succeeded, for example, in the refinement of the crystal structures with following characteristics:

1: $\mathrm{a}=61.88, \mathrm{~b}=68.87, \mathrm{c}=69.66 \AA, \mathrm{V}=296829 \AA^{3}, F d d d$, 370 unique non-H positions, $\mathrm{d}>0.83 \AA, \mathrm{R} 1=0.15$

2: $a=43.12, b=42.13, c=43.28 \AA, \beta=92.31^{\circ}, V=78555$ $\AA^{3}, C 2 / c, 585$ non-H's, d $>0.83 \AA$, R $1=0.08$

3: $a=46.4, b=31.92, c=91.33 \AA, \beta=97.44^{\circ}, V=$ $134142 \AA^{3}, C 2 / c, 762$ non-H's, d>0.98 $\mathrm{A}, \mathrm{R} 1=0.13$

[1] E. V. Peresypkina et al (2014), Z. Kristallogr., 229, 735 , and references therein.

[2] F. Dielmann et al (2015), Chem.-A Eur. J., DOI: 10.1002/chem.201500692. 